J. Andersen (ed.), Reports on Astronomy, Vol. XXIVA, 77-84.

(C) 2000 IAU. Printed in the United States of America.

\title{
COMMISSION 49: INTERPLANETARY PLASMA AND HELIOSPHERE
}

\author{
(PLASMA INTERPLANETAIRE ET HELIOSPHERE)
}

\author{
PRESIDENT: F. Verheest \\ VICE-PRESIDENT: M. Vandas \\ ORGANIZING COMMITTEE: B. Buti, N.F. Cramer, M. Dryer, S.R. Habbal, \\ J.V. Hollweg, M.C.E. Huber, M. Kojima \& H. Ripken
}

\section{PREFACE}

\section{F. VERHEEST}

President of the Commission

Sterrenkundig Observatorium, Universiteit Gent, B-9000 Gent, Belgium

In the last decade the triennial reports from Commission 49 have covered various topics like (nonlinear) plasma processes, magnetohydrodynamic phenomena and flows in the heliosphere, solar wind composition, transient events in, and latitudinal dependencies of, the heliosphere, interstellar gas flow through the interface region, kinetic versus magnetohydrodynamic theory in heliospheric plasmas and charged dust in space plasmas. Continuing the tradition of summarizing specific aspects to give astronomers outside our own specialty a flavour of our field, we now address recent advances in understanding coronal mass ejections in interplanetary space and the inner heliospheric solar wind under quiet and perturbed conditions. We owe a great debt of gratitude to the eminent contributors for their valiant efforts in writing these succinct but clear reports and guiding us through the recent literature.

\section{CORONAL MASS EJECTIONS IN INTERPLANETARY SPACE (ICMEs)}

\author{
M. VANDAS ${ }^{1}$ and M. DRYER ${ }^{2}$
}

1 Astronomical Institute, Czech Academy of Sciences, Prague, Czech Republic

${ }^{2}$ NOAA Space Environment Center, Boulder, Colorado, USA

\subsection{Introduction}

Coronal mass ejections are huge plasma ejections from the upper solar atmosphere (corona) which then travel through the interplanetary space. CMEs carry complex solar magnetic fields and they are thought to be a consequence of a global reconfiguration of coronal magnetic fields (e.g., Weiss et al. 1996). Although discovered in the 1970s and since then intensively studied, the most basic questions still remain unanswered: What is the primary trigger of ejections? What is their true three dimensional shape and magnetic field configuration? What is their fate in interplanetary space? This article will cover mainly new findings on CMEs in interplanetary space during the last four years and, briefly, CME initiation and their characteristics near the Sun. Reference will be made to the growing importance of interplanetary scintillations (IPS) to the study of propagating disturbances via this remote, all-sky, radio sensing technique. We will also comment on the status of three-dimensional magnetohydrodynamic (3D MHD) modeling of solar-generated interplanetary disturbances and some pioneering studies that use this approach for space weather 
prediction efforts. The acronym, ICME, has been suggested (Dryer, 1994) as a distinction between coronagraph (near-Sun) and interplanetary observations. Studies of ICMEs were enabled by an abundance of in situ measurements from older spacecraft such as Helios 1-2, ISEE 1-3, IMP 8, Voyager 1-2, as well as from recent ones such as Ulysses, Wind, SOHO, and ACE.

The first conference especially devoted to CMEs, the Chapman Conference "Coronal Mass Ejections: Causes and Consequences" was held in Bozeman, Montana, USA, in 1996 (the proceedings have been published as Geophysical Monograph 99). Other conferences dealing with CMEs to a larger extent were SOLTIP III Symposium (Beijing, Peoples' Republic of China, 1996) and Solar Wind 9 Conference (Nantucket, Massachusetts, 1998).

\subsection{Interplanetary signatures of CMEs}

There are no definite rules for identification of solar ejecta in solar wind measurements and this task needs some observer's experience. Many signatures were described in the literature, the most common being counterstreaming of suprathermal electrons, low proton temperature, anomalously low or high electron temperature, stronger magnetic field, smooth magnetic field rotation, low magnetic field variance, helium abundance enhancement, and unusual ionization states. A single ejection usually exhibits only a few of these signatures and, moreover, they only partly overlap in time or even do not overlap at all (Bravo, BlancoCano, \& Chi-Cerrito 1997; Crooker, Gosling, \& Kahler 1998a; Goldstein, Neugebauer, \& Clay 1998). This suggests that CMEs are structured on global scales and their plasma comes from various coronal regions. This is also supported by Gloeckler et al. (1999) who found from ionization states that CME plasma is a mixture from cold and hot coronal regions. Richardson, Farrugia, \& Cane (1997) studied the electron temperature $\left(T_{e}\right)$ in ejecta and found that in some parts of the ejecta $T_{e}$ may be enhanced and depressed in another. $T_{e}$ anticorrelates with the density and is significantly enhanced over the proton temperature $\left(T_{p}\right)$. This is most striking in magnetic clouds where $T_{e} / T_{p} \approx 7$ (Sittler \& Burlaga 1998). Magnetic clouds are a well-defined subset of CMEs, recognized mainly by the stronger magnetic field and its smooth rotation. They are also distinguished by enhanced $\mathrm{O}^{7+} / \mathrm{O}^{6+}$ while other CMEs have this ratio similar to the ambient solar wind (Henke et al. 1998). Gonzalez et al. (1998) found a correlation between the magnetic cloud peak field strength and peak velocity. Traces of an erupting prominence in the interplanetary space were found behind a magnetic cloud as a density pulse (Burlaga et al. 1998; Gopalswamy et al. 1998).

\subsection{CMEs and remote sensing via interplanetary scintillations}

The radio astronomical technique of remote sensing, via IPS, of propagating disturbances through the solar wind has made important advances since the last Report. These advances were made possible by the cooperation of the radio observatories in India and Japan. Both observatories (Ooty and Toyokawa) operate at $327 \mathrm{MHz}$ and can, together, increase the number of daily-observed radio sources, thereby increasing the number of all-sky grid points. The large, single, steerable, parabolically-cylindrical antenna at Ooty obtains solar wind speed (via parameterized, density fluctuation power spectra) as well as relative density fluctuating power levels at the closest approach of the line-of-sight to the Sun (the so-called P-point). Similar, but smaller, antennae are located at Toyokawa and three other locations in Japan. Here, the solar wind speed is found by cross-correlations of moving density fluctuations across the array baselines. Results of IPS studies (Janardhan et al. 1996; Kojima et al. 1998; Tokumaru et al. 1999; Ananthakrishnan et al. 1999) demonstrated the close associations of specific solar flares and their CMEs and shocks that continued into interplanetary space. This approach has the advantage of showing all-sky maps, using many radio sources, of the velocity and density fluctuation parameter, $g$, which is the ratio of power integrated along the l-o-s (peaking at the P-point) to that for the same radio source at the same elongation under long-term conditions, usually 12 months at or near solar minimum. Another important advantage is that the out-of-ecliptic sky can be mapped. 
The disadvantages, of course, are lack of IMF conditions and, for just the two sites in Japan and India, a somewhat coarse temporal resolution.

This last disadvantage, however, can be overcome in a sense by devoting the radio telescope to a single source search, with excellent temporal resolution, for a predicted shock and its ICME as has been done by Gothoskar \& Rao (1999). In such cases, the P-point may be thought of as a virtual spacecraft during its daily scan of a single radio source.

\subsection{Shape and structure of CMEs}

Global shape and structure of CMEs are poorly known. Neugebauer, Goldstein, \& Goldstein (1997) reported long lasting periods of radial magnetic field within the trailing part of fast CMEs which may be a signature of legs in a loop-like body. More is known about magnetic clouds which are treated as large loops with helical magnetic field (flux ropes) rooted in the corona. Marubashi (1997) suggested that the loop is deformed due to the solar rotation which can explain some peculiar observations of magnetic clouds. Crooker et al. (1998a) suggested that the deformation may cause a spacecraft to cross a cloud two times. However the recent findings indicate that magnetic cloud structure will be more complicated, that they probably consist of two or more flux tubes which are intertwined (Vandas 1998; Osherovich, Fainberg, \& Stone 1999). A complicated relationship between magnetic clouds and sector boundaries was studied by Crooker et al. (1998a, 1998b).

\subsection{MHD modeling of CMEs}

CMEs are usually modeled as a pressure pulse near the Sun. A number of 3D MHD parametric studies were recently performed with the axisymmetrical assumption; that is, partial derivatives of all physical parameters (velocity, IMF, density, and temperature of a single fluid) in the heliolongitudinal direction were taken to be zero. It was found by Odstrčil, Dryer, \& Smith (1996) that the heliospheric current/plasma sheet (HCS/HPS) plays a very significant role in the attenuation and propagation of any shocks that propagate within it or that attempt to cross it. The axisymmetric assumption was relaxed by Odstrčil \& Pizzo (1999). These workers showed the consequences following solar-generated pressure pulses (initialized at $0.1 \mathrm{AU}$ and referred-to as $\mathrm{CMEs}$ ) as the subsequent disturbances propagated through a tilted HCS/HPS or from either side, eastward or westward, of this discontinuity. Using a wedge-like HCS/HPS patterned after the Ulysses' solar minimum observations, they showed vast differences in the distortions of the ICMEs.

A number of 3D MHD applications was also directed to epochs of specific events. Odstrčil, Dryer, \& Smith (1998) focused on the series of the March 1991 solar flares. They started from the solar source surface and used an empirical approach to extrapolate to $0.1 \mathrm{AU}$ where the 3D MHD computation was then started. A limited comparison of solar wind speed (and shocks) was made in the ecliptic plane with Ulysses, Galileo, IMP-8, and Pioneer-Venus-Orbiter observations. The non-flaring scenario of a vast, erupting, southern hemisphere helmet streamer on 14 April 1994 was simulated in 3D by Dryer, Wu, \& Smith (1997). Initialization was suggested by a temporally- and spatially-varying density input (hence pressure) observed by Yohkoh's SXT's emission measure and line ratio observations. The result provided excellent shock arrival times at both Earth and at Ulysses which was located at S60E30.

Magnetic clouds are modeled as toroidal flux ropes. Chen (1996) extended his analytical model of a coronal loop to its evolution in the interplanetary space and found a quite good agreement with observations (Chen et al. 1997). Wu et al. $(1997,1999)$ and Wu \& Guo (1999), through an MHD modeling procedure, followed a toroidal flux rope from the inner corona to the interplanetary space. Wu et al. (1999) showed the interplanetary manifestation of the original white-light CME's three-part structure: the shock, ICME, and magnetic cloud that resembles the 6-12 January 1997 Sun-Earth connection event. Vandas et al. (1997) studied an interaction of a magnetic cloud with a traveling shock wave; this interaction increases magnetic cloud geoeffectiveness. 
It is suggested that many of these 3D MHD exercises are required in order to validate the background conditions, initialization, and in situ output parameters for the space weather objective.

\subsection{Prediction of geoeffective CMEs}

Magnetic clouds are potentially geoeffective because of their stronger magnetic field and long lasting southward magnetic field component which may trigger a geomagnetic storm. The relationship between erupting solar filaments and magnetic clouds was studied by Bothmer \& Rust (1997). Zhao \& Hoeksema (1997, 1998) found a correlation between axial magnetic cloud directions and their duration and magnetic field intensities, which may be used for predictions. Chen, Cargill, \& Palmadesso (1997) elaborated a prediction scheme for a magnetic cloud advent using real time solar wind data from spacecraft in the Earth's libration point L1 (such as Wind or ACE).

\section{References}

Ananthakrishnan, S., Tokumaru, M., Kojima, M., Balasubramanian, V., Janardhan, P., Manoharan, P.K., \& Dryer, M. 1999, in AIP Conf. Proceedings 471, Solar Wind Nine, ed. S.R. Habbal, R. Esser, J.V. Hollweg, \& P.A. Isenberg (Woodbury: AIP), 321

Bothmer, V., \& Rust, D.M. 1997, in Geophysical Monograph 99, Coronal Mass Ejections, ed. N. Crooker, J.A. Joselyn, \& J. Feynman (Washington: AGU), 139

Bravo, S., Blanco-Cano, X., \& Chi-Cerrito, K. 1997, Adv. Space Res., 20, 107

Burlaga, L., Fitzenreiter, R., Lepping, R., Ogilvie, K., Szabo, A., Lazarus, A., Steinberg, J., Gloeckler, G., Howard, R., \& Michels, L. 1998, JGR, 103, 277

Chen, J. 1996, JGR, 101, 27499

Chen, J., Howard, R.A., Brueckner, G.E., Santoro, R., Krall, J., Paswaters, S.E., St. Cyr, O.C., Schwenn, R., Lanny, P., \& Simnett, G.M. 1997, ApJ Lett., 490, L191

Chen, J., Cargill, P.J., \& Palmadesso, P.J. 1997, JGR, 102, 14701

Crooker, N.U., Gosling, J.T., Kahler, S.W. 1998a, JGR, 103, 301

Crooker, N.U., McAllister, A.H., Fitzenreiter, R.J., Linker, J.A., Larson, D.E., Lepping, R.P., Szabo, A., Steinberg, J.T., Lazarus, A.J., Mikic, Z., \& Lin, R.P. 1998b, JGR, 103,26859

Dryer, M. 1994, Space Sci. Rev., 67, 363

Dryer, M., Wu, C.-C., Smith, Z.K. 1997, JGR, 102, 14065

Gloeckler, G., Fisk, L.A., Hefti, S., Schwadron, N.A., Zurbuchen, T.H., Ipavich, F.M., Geiss, J., Bochsler, P., \& Wimmer-Schweingruber, R.F. 1999, GRL, 26, 157

Goldstein, R., Neugebauer, M., \& Clay, D. 1998, JGR, 103, 4761

Gonzalez, W.D., Clúa de Gonzalez, A.L., Dal Lago, A., Tsurutani, B.T., Arballo, J.K., Lakhina, G.S., Buti, B., \& Ho, C.M. 1998, GRL, 25, 963

Gopalswamy, N., Hanaoka, Y., Kosugi, T., Lepping, R.P., Steinberg, J.T., Plunkett, S., Howard, R.A., Thompson, B.J., Gurman, J., Ho, G., Nitta, N., \& Hudson, H.S. 1998, GRL, 25, 2485

Gothoskar, P., \& Rao, A.P. 1999, Solar Phys., 185, 361

Henke, T., Woch, J., Mall, U., Livi, S., Wilken, B., Schwenn, R., Gloeckler, G., von Steiger, R., Forsyth, R.J., \& Balogh, A. 1998, GRL, 25, 3465

Janardhan, P., Balasubramanian, V., Ananthakrishnan, S., Dryer, M., Bhatnagar, A., \& McIntosh, P. 1996, Solar Phys., 166, 379

Kojima, M., Asai, K., Jackson, B.V., Hick, P.L., Tokumaru, M., Watanabe, H., \& Yokobe, A. 1998, in Proceedings of the Third SOLTIP Symposium, Advances in Solar 
Connection with Transient Interplanetary Phenomena, eds. X.S. Feng, F.S. Wei, \& M. Dryer (Beijing: Int'l. Acad. Publ.), 207

Marubashi, K. 1997, in Geophysical Monograph 99, Coronal Mass Ejections, ed. N. Crooker, J.A. Joselyn, \& J. Feynman (Washington: AGU), 147

Neugebauer, M., Goldstein, R., \& Goldstein, B.E. 1997, JGR, 102, 19743

Odstrčil, D., Dryer, M., \& Smith, Z. 1996, JGR, 101, 19973

Odstrčil, D., Dryer, M., \& Smith, Z. 1998, in Proceedings of the Third SOLTIP Symposium, Advances in Solar Connection with Transient Interplanetary Phenomena, eds. X.S.

Feng, F.S. Wei, \& M. Dryer (Beijing: Int'l. Acad. Publ.), 191

Odstrčil, D., \& Pizzo, V.J. 1999, JGR, 104, 493

Osherovich, V.A., Fainberg, J., \& Stone, R.G. 1999, GRL, 26, 401

Richardson, I.G., Farrugia, C.J., \& Cane, H.V. 1997, JGR, 102, 4691

Sittler Jr., E.C., \& Burlaga, L.F. 1998, JGR, 103, 17447

Tokumaru, M., Kojima, M., Ohmi, T., Kondo, T., \& Hakamada, K. 1999, in AIP Conf. Proceedings 471, Solar Wind Nine, ed. S.R. Habbal, R. Esser, J.V. Hollweg, \& P.A. Isenberg (Woodbury: AIP), 313

Vandas, M., Fischer, S., Dryer, M., Smith, Z., Detman, T., \& Geranios, A. 1997, JGR, 102, 22295

Vandas, M. 1998, in Proceedings of the Third SOLTIP Symposium, Advances in Solar Connection with Transient Interplanetary Phenomena, eds. X.S. Feng, F.S. Wei, \& M. Dryer (Beijing: Int'l. Acad. Publ.), 259

Weiss, L.A., Gosling, J.T., McAllister, A.H., Hundhausen, A.J., Burkepile, J.T., Phillips, J.L., Stray, K.T., \& Forsyth, R.J. 1996, A\&A, 316, 384

Wu, S.T., Guo, W.P., Andrews, M.D., Brueckner, G.E., Howard, R.A., Koomen M.J., Korendyke, C.M., Michels, D. J., Moses, J.D., Socker, D.G., Dere, K.P., Lamy, P.L., Llebaria, A., Bout, M.V., Schwenn, R., Simnett, G.M., Bedford, D.K., \& Eyles, C.J. 1997, Sol. Phys., 175, 719

Wu, S.T., \& Guo, W.P. 1999, J. Atm. Solar-Terr. Phys., 61, 109

Wu, S.T., Guo, W.P., Michels, D.J., \& Burlaga, L.F. 1999, JGR, 104, 14789.

Zhao, X.P., \& Hoeksema, J.T. 1997, GRL, 24, 2965

Zhao, X.P., \& Hoeksema, J.T. 1998, JGR, 103, 2077

\section{INNER HELIOSPHERIC SOLAR WIND}

M. KOJIMA ${ }^{1}$ and S. ANANTHAKRISHNAN ${ }^{2}$

1 Solar-Terrestrial Environment Laboratory, Nagoya University, 3-13 Honohara, Toyokawa 442-8507, Japan

${ }^{2}$ NCRA-TIFR, Pune University Campus, Pune 411007, India

\section{Part A: INNER HELIOSPHERIC SOLAR WIND UNDER QUIET CONDITIONS}

\subsection{Interplanetary scintillation}

Since the discovery of the interplanetary scintillation (IPS) phenomenon, IPS observation techniques and data analysis methods have been developed to obtain high reliabilities in radio remote sensing observations. Recently developed techniques help in deconvolving the line-of-sight (los) integration effect inherent in IPS observations and retrieve unbiased solar wind speeds with high spatial resolution. These techniques use the long-base-line IPS observation method (Grall et al. 1996) and a computer assisted tomography (CAT) analysis (Asai et al. 1998; Jackson et al. 1998; Kojima et al. 1998). For obtaining high temporal 
resolution from IPS and for detecting short time scale activity, continuous tracking of radio sources has also been carried out (Gothoskar and Rao, 1996, 1999).

\subsection{Acceleration of high-speed solar wind}

Extensive investigations of solar wind acceleration have been made, and rapid acceleration phenomena of the high-speed solar wind have been discovered within 10 solar radii (Grall et al. 1996; Breen et al. 1996) with great impact on solar wind acceleration theories (e.g., Feldman et al. 1997; Esser et al. 1997; McKenzie \& Axford 1997). Further confirmation of the rapid acceleration phenomena come from the work of Shishov \& Tokumaru (1996), Tokumaru et al. (1997), Yamauchi et al. (1998), Kojima et al. (1998), Breen et al. (1999) and Tokumaru et al. (1999a).

\subsection{Global structure of the solar wind in three dimensions}

The line-of-sight integration free IPS observations (Breen et al. 1996; Grall et al. 1996; Kojima et al. 1998) have confirmed the bimodal speed structure consisting of distinct fast $(\sim 800 \mathrm{~km} / \mathrm{s})$ and slow $(\sim 400 \mathrm{~km} / \mathrm{s})$ components, which had been obtained long before the Ulysses spacecraft observations by the IPS. Evolution of the bimodal structure and compression regions at a leading edge of a fast stream have been investigated by dualfrequency ranging measurements of Ulysses radio signals (Bird et al. 1996) and by IPS observations (Breen et al. 1999).

\subsection{Origin and acceleration of low-speed wind}

The origin of a low-speed streamer has been revealed by making direct observations of its stalk structure by the radio signal occultation method (Woo 1997; Woo \& Martin 1997; Habbal et al. 1997) and by analyzing the relation with active regions (Jackson et al. 1998; Kojima et al. 1999). The acceleration profile of low-speed streams has been studied by measuring the inner scale length (Yamauchi et al. 1998) and by dual-frequency ranging data analysis (Pätzold et al. 1997).

\subsection{Source regions of the solar wind}

Solar wind source regions in corona have been studied by combining IPS and other ground based coronal observations. The tomography analysis of IPS and coronagraph data has obtained clear anti-correlation between the solar wind speed in interplanetary space and electron density in the lower corona (Yokobe et al. 1998).

\subsection{Microscale fluctuations}

Properties of density and magnetic field fluctuations have been studied at distances from a near sun region to $1 \mathrm{AU}$ by various radio propagation observations such as intensity scintillation, spectral broadening, phase scintillation, and Faraday rotation measurements using spacecraft signals and natural radio sources (Andreev et al. 1996; Pätzold et al. 1996; Yamauchi et al. 1996; Andreev et al. 1997; Karl et al. 1997; Asai et al. 1998).

\section{PART B: SOLAR WIND UNDER DISTURBED CONDITIONS}

When the solar wind is disturbed due to transient activities like solar flares, coronal mass ejections (CMEs) or transient coronal holes, the disturbances propagate into the heliosphere. These interplanetary disturbances (IPD's) can be detected as enhanced scintillation by IPS.

The spatial and dynamical distribution of a large number (about 740) of IPD's within the distance range 0.25 - 1 AU have been studied (Manoharan 1997), based on scintillation measurements during 1986 and 1991. Their helio-latitudinal distribution indicates that near the solar minimum IPD's are confined to the ecliptic region, whereas, when the activity 
increases, the distribution spreads to the poles. This is consistent with the distribution of large scale coronal magnetic features and the latitude drift of coronal mass ejections. These disturbances show a wide range of speeds, with a global average speed of about $450 \mathrm{~km} / \mathrm{s}$. More than $80 \%$ of the disturbances are associated with the active prominences on the sun, which are suggested to be the source of CMEs.

The occurrence rate of disturbances also shows a dependence on the solar cycle. A nearly three times higher rate has been observed in 1990 than in the period of minimum activity during 1986-87 (Manoharan 1996). Such variation is also true for the CME rates. These results indicate that the location of the current sheet (flat along the sun's equator at the solar minimum and warped with complicated structure at the maximum) plays an important role in the formation of the solar drivers of interplanetary transients at different latitudes (Manoharan 1998a, 1998b).

Thirty-three fast IPD's with velocities ranging from 600 to $1400 \mathrm{~km} / \mathrm{s}$ have been studied with high temporal resolution. By modeling them using a two-component model (Gothoskar and Rao, 1996, 1999), the geometry, energy and mass associated with these disturbances have been estimated. The average mass and kinetic energy were found to be $5.3 \times 10^{16}$ gm and $3.5 \times 10^{34} \mathrm{ergs}$, respectively. Average opening angle and width was $42 \mathrm{deg}$ and $8 \times 10^{6} \mathrm{~km}$. The distribution of mass and energy was in agreement with the distribution of less energetic CMEs. Further, mass ejections with higher mass were found having higher velocity. It was also found that $80 \%$ of the above sample was associated with long duration x-ray events (LDXE) and CME's and $50 \%$ of them produced geomagnetic disturbances (Gothoskar and Rao, 1999).

By using a set of spatially well distributed compact radio sources as a rapidly movable 'picket fence' several traveling IPD's have been monitored (Ananthakrishnan, 1996, Janardhan et al., 1996, 1997, Balasubramanian et al. 1998). Unlike the earlier methods used by the Cambridge group, both the relative scintillation index $g$, as well as the velocity estimated based on a single station, have been used to reduce the ambiguities. In addition, by combining the data from IPS observatories in India and Japan, it has been shown that, in the case of energetic solar flares, there is almost a one-to-one correspondence between energetic flare associated shocks and transient IPD's which can be tracked all the way from close to the Sun to near Earth (Ananthakrishnan et al., 1999).

Frank Verheest

President of the Commission

\section{References}

Ananthakrishnan, S., 1996, Astrophys. Sp. Sci., 243, 215

Ananthakrishnan, S., M. Tokumaru, M. Kojima, V. Balasubramanian, P. Janardhan, P.K. Manoharan, \& M. Dryer, 1999, in Solar Wind Nine, AIP Conf. Proc. 471, Nantucket Island, Massachusetts, USA, 5-9 Oct., 313

Andreev, V.E., A.I. Efimov, L.N. Samoznaev, \& M.K. Bird, 1996, in Solar Wind Eight, AIP Conf. Proc. 382, D. Winterhalter et al. (eds.), AIP Press, Woodbury, NY/USA, 34

Andreev, V.E., A.I. Efimov, L.N. Samoznaev, I.V. Chashei, \& M.K. Bird, 1997, Solar Phys., 176,387

Asai, K., M. Kojima, M. Tokumaru, A. Yokobe, B.V. Jackson, P. Hick, \& P.K. Manoharan, 1998, J. Geophys. Res., 103, 1991

Balasubramanian, V., P. Janardhan, R. Srivatsan, \& S. Ananthakrishnan, 1998b, Proc. III SOLTIP Symp., Eds. X.S. Feng, F.S. Wu and M. Dryer, International Academic Publishers, 249

Bird, M.K., M. Pätzold, P. Edenhofer, S.W. Asmar, \& T.P. McElrath, 1996, A\&A, 316, 441 
Breen, A.R., W.A. Coles, R.R. Grall, M.T. Klinglesmith, J. Markkanen, P.J. Moran, B. Tegid, \& P.J.S. Williams, 1996, Ann. Geophysicae, 14, 1235

Breen, A.R., Z. Mikic, J.A. Linker, A.J. Lazarus, B.J. Thompson, D.A. Biesecker, P.J. Moran, C.A. Varley, P.J.S. Williams, \& A. Lecinski, 1996, J. Geophys. Res., 104, 9847

Esser, R., S.R. Habbal, W.A. Coles, \& J.V. Hollweg, 1997, J. Geophys. Res., 102, 7063

Feldman, W.C., S.R. Habbal, G. Hoogeveen, \& Y.-M. Wang, 1997, J. Geophys. Res., 102, 26905

Gothoskar, P. \& A.P. Rao, 1996, Astrophys. Sp. Sci., 243, 225

Gothoskar, P. \& A.P. Rao, 1999, Solar Phys., 185, 361

Grall, R.R., W.A. Coles, M.T. Klinglesmith, A.R. Breen, P.J.S. Williams, J. Markkanen, \& R. Esser, 1996, Nature, 379, 429

Habbal, S.R., R. Woo., S. Fineschi, R. O'Neal, J. Kohl, G. Noci, \& C. Korendyke, 1997, ApJ, 489, L103

Jackson, B.V., P.L. Hick, M. Kojima, \& A. Yokobe, 1998, J. Geophys. Res., 103, 12049

Janardhan, P., V. Balasubramanian, S. Ananthakrishnan, M. Dryer, A. Bhatnagar, \& P.I. McIntosh, 1996, Solar Phy., 166, 379

Janardhan, P., V. Balasubramanian, \& S. Ananthakrishnan, 1997, Proc. 31st ESLAB symp., ESTEC, Noordwijk, The Netherlands, 22-25 Sep., 1997, 177

Karl, J., M. Pätzold, \& M.K. Bird, 1997, Geophys. Res. Lett., 24, 2881

Kojima, M., M. Tokumaru, H. Watanabe, A. Yokobe, K. Asai, B.V. Jackson, \& P.L. Hick, 1998, J. Geophys. Res., 103, 1981

Kojima, M., K. Fujiki, T. Ohmi, M. Tokumaru, A. Yokobe, \& K. Hakamada, 1999, J. Geophys. Res., 104, 16993

Manoharan, P.K., 1996, Astrophys. Sp. Sci., 243, 221

Manoharan, P.K., 1997, Geophys. Res. Lett., 24, 2623

Manoharan, P.K., 1998a, Bull. Astr. Soc. India, 26, 211

Manoharan, P.K., 1998b, Proc. III SOLTIP Symp., Eds. X.S. Feng, F.S. Wu and M. Dryer, International Academic Publishers, 249

McKenzie, J.F., \& W.I. Axford, 1997, Geophys. Res. Lett., 24, 2877

Pätzold, M., J. Karl, \& M.K. Bird, 1996, A\&A, 316, 449

Pätzold, M., B.T. Tsurutani, \& M.K. Bird, 1997, J. Geophys. Res., 102, 24151

Shishov, V.I., \& M. Tokumaru, 1996, J. Geomag. Geoelectr., 48, 1461

Tokumaru, M., H. Mori, T. Tanaka, T. Kondo, \& H. Takaba, 1997, Proc. STPW '96, 138

Tokumaru, M., M. Kojima, T. Ohmi, T. Kondo, \& K. Hakamada, 1999a, in Solar Wind Nine, AIP Conf. Proc. 471, Nantucket Island, Massachusetts, USA, 5-9 Oct., 313

Woo, R., 1997, Geophys. Res. Lett., 24, 97

Woo, R., \& J.M. Martin, 1997, Geophys. Res. Lett., 24, 2535

Yamauchi, Y., M. Kojima, M. Tokumaru, H. Misawa, H. Mori, T. Tanaka, H. Takaba, T. Kondo, \& P.K. Manoharan, 1996, J. Geomag. Geoelectr., 48, 1201

Yamauchi, Y., M. Tokumaru, M. Kojima, P.K. Manoharan, \& R. Esser, 1998, J. Geophys. Res., 103, 6571

Yokobe, A., T. Ohmi, K. Hakamada, M. Kojima, M. Tokumaru, B.V. Jackson, P.L. Hick, \& S. Zidowitz, 1998, in Solar Wind Nine, AIP Conf. Proc., Nantucket Island, Massachusetts, USA, 5-9 Oct., 565 